\title{
Second-Order Multiagent Systems with Event-Driven Consensus Control
}

\author{
Jiangping Hu, Yulong Zhou, and Yunsong Lin \\ School of Automation Engineering, University of Electronic Science and Technology of China, Chengdu 610054, China \\ Correspondence should be addressed to Jiangping Hu; hjp_lzu@163.com
}

Received 27 November 2012; Accepted 3 April 2013

Academic Editor: Pedro M. Lima

Copyright (C) 2013 Jiangping Hu et al. This is an open access article distributed under the Creative Commons Attribution License, which permits unrestricted use, distribution, and reproduction in any medium, provided the original work is properly cited.

Event-driven control scheduling strategies for multiagent systems play a key role in future use of embedded microprocessors of limited resources that gather information and actuate the agent control updates. In this paper, a distributed event-driven consensus problem is considered for a multi-agent system with second-order dynamics. Firstly, two kinds of event-driven control laws are, respectively, designed for both leaderless and leader-follower systems. Then, the input-to-state stability of the closed-loop multiagent system with the proposed event-driven consensus control is analyzed and the bound of the inter-event times is ensured. Finally, some numerical examples are presented to validate the proposed event-driven consensus control.

\section{Introduction}

Recently, synthesis and analysis of multi-agent systems have drawn great attention in many disciplines, such as mathematics, physics, computer science, systems biology, engineering, and social science. Roughly speaking, multi-agent systems are a class of networked dynamic systems consisting of a group of autonomous agents, which interact with each other locally and achieve an emergence behavior over a communication network. The controlled multi-agent systems have a broad range of applications including flocking and swarming in animal groups, vehicle formation, satellite reconfiguration, and unmanned aerial vehicles for rescue and surveillance.

Consensus problems have a long history originated from management science and statistics in 1960s [1]. In the context of multi-agent systems, consensus generally means to reach an agreement regarding a certain quantity of interest that depends on the state of all agents [2]. In the literature of consensus control of multi-agent systems, many works have been focused on an important issue, that is, to investigate the coordination behavior of agents governed by different order dynamics. Especially, first-order multi-agent systems are extensively considered as a representative multi-agent consensus model in, for example, [3-8] and references therein. More recently, the consensus problems of multi-agent systems with second-order dynamics (e.g., [9-14]) and high order dynamics (e.g., [15-19]) have been paid much attention, which is mainly because in many real applications mass-point models are invalid for agents and more complex dynamics should be considered. Generally, the dynamics of a secondorder multi-agent system is described by a second-order differential equation or difference equation, which contains both the position and the velocity information.

One potential application of multi-agent control is to equip each autonomous agent with a small embedded microprocessor to collect information from neighboring agents for actuating the controller updates. However, micro-processors are generally resource- and energy-limited [20], which requires a time- or an event-triggered scheduling strategy to update the control. A time-triggered update scheduling involves sampling at predefined time instances while an event-triggered one executes the control task whenever a certain error becomes large compared with the state norm. Time-triggered consensus problems were studied in [14, $21,22]$ via data-sampled method. However, event-triggered strategies seem more favorable in applications. A distributed event-triggered control was considered for a first-order multi-agent system in [23-25]. Up to date, there are few contributions devoted to designing an event-triggered consensus control for multi-agent systems with second-order dynamics.

In this paper, we consider an event-driven consensus problem of a second-order leaderless and leader-follower 
multi-agent system with a fixed directed communication network. Firstly, the event-driven consensus problem is formulated. Secondly, an event-driven consensus control is designed for each agent to achieve consensus. Then the closed-loop multi-agent system is proven to be input-to-state stable with respect to the measurement error and, simultaneously, a positive lower bound is found for the event-time between two consecutive actuation updates.

Throughout this paper, the following notations are used. $I_{n \times n}$ denotes an $n \times n$ identity matrix; $0_{n \times m}$ denotes an $n \times m$ zero matrix; $\operatorname{col}(\cdot)$ denotes a column vector; $\mathbf{1}_{n}=$ $\operatorname{col}(1, \ldots, 1) \in \mathbb{R}^{n}$. The norm of a vector $x \in \mathbb{R}^{n}$ is defined as $\|x\|=\sqrt{x^{T} x}$. The spectral norm of matrix $A \in \mathbb{R}^{m \times n}$ is defined as $\|A\|=\max _{1 \leq i \leq n} \sqrt{\lambda_{i}}$, where $\lambda_{i}$ are eigenvalues of $A^{T} A$. For a matrix $A \in \mathbb{R}^{n \times n}, \lambda_{\text {min }}(A)$ and $\lambda_{\text {max }}(A)$ denote, respectively, its minimal and maximal eigenvalues.

\section{Preliminaries and Problem Formulation}

2.1. Some Preliminaries. Let $\mathscr{G}=(\mathscr{V}, \mathscr{E}, A)$ be a weighted directed graph with a set of vertices $\mathscr{V}=\{1, \ldots, n\}$, a set of arcs $\mathscr{E} \subseteq \mathscr{V} \times \mathscr{V}$, and a weighted adjacency matrix $A=\left[a_{i j}\right] \epsilon$ $\mathbb{R}^{n \times n}$. In the mapping of graph $\mathscr{G}$ to the interconnection topology of a multi-agent system, vertex $i \in \mathscr{V}$ represents agent $i$, and $\operatorname{arc}(i, j)$, which starts from vertex $i$ and ends on vertex $j$, is in $\mathscr{E}$ if and only if agent $i$ can receive information from agent $j$. In this case, agent $j$ is called a neighbor of agent $i$, and, accordingly, $\mathcal{N}_{i}=\{j \mid(i, j) \in \mathscr{E}\}$ denotes the neighboring set of agent $i$. The element $a_{i j}$ in the adjacency matrix $A$ is associated with the $\operatorname{arc}(i, j)$, that is, $a_{i j}>0$ if and only if $(i, j) \in \mathscr{E}$. Moreover, we assume that $a_{i i}=0$ for all $i \in \mathscr{V}$. When a single leader-agent is involved in the multi-agent systems, a vertex 0 is added to represent the leader-agent and the interconnection topology is denoted by $\overrightarrow{\mathscr{G}}=(\overrightarrow{\mathscr{V}}, \overrightarrow{\mathscr{E}})$ with $\overrightarrow{\mathscr{V}}=\{0,1,2, \ldots, n\}$ and $\overrightarrow{\mathscr{E}}=\{(i, j) \mid i, j \in \overrightarrow{\mathscr{V}}\}$.

Definition 1 (see [26]). A path from vertex $i$ to vertex $j$ is a sequence of $\operatorname{arcs}\left(i_{0}, i_{1}\right),\left(i_{1}, i_{2}\right), \ldots,\left(i_{l-1}, i_{l}\right)$ in the directed graph $\mathscr{G}$ with distinct vertexes $i_{k}, k=0,1, \ldots, l$ and $i_{0}=i$, $i_{l}=j$. A directed graph $\mathscr{G}$ is strongly connected if there exists a path from vertex $i$ to vertex $j$, for every $i, j \in \mathscr{V}$.

Definition 2 (see [26]). Vertex $j$ is said to be reachable from vertex $i$ if there exists a path from vertex $i$ to vertex $j$ in the directed graph $\mathscr{G}$. Vertex $j$ is globally reachable if there exists a path from every other vertex to vertex $j$ in $\mathscr{G}$.

According to Definitions 1 and 2, a directed graph $\mathscr{G}$ is strongly connected if and only if each vertex in $\mathscr{G}$ is globally reachable, which shows that the global reachability of a directed graph is much weaker than the strong connectedness.

A diagonal matrix $D=\operatorname{diag}\left(d_{1}, \ldots, d_{n}\right) \in \mathbb{R}^{n \times n}$ is a degree matrix of $\mathscr{G}$, whose diagonal elements $d_{i}=\sum_{j \in \mathcal{N}_{i}} a_{i j}$ for $i=1, \ldots, n$. Then the Laplacian matrix of a weighted directed graph is defined as

$$
L=D-A \text {. }
$$

The next lemma shows an important property of Laplacian matrix $L$ associated with directed graph $\mathscr{G}$.

Lemma 3 (see [4]). Laplacian matrix L has least one zero eigenvalue with $\mathbf{1}_{n}$ as its eigenvector, and all the non-zero eigenvalues of $L$ have positive real parts. $L$ has a simple zero eigenvalue if and only if $\mathscr{G}$ has a globally reachable vertex.

In the leader-follower consensus literature, it is always assumed that the leader-agent is self-active, that is, the leader does not need information feedback from other agents and thus, the adjacency coefficients $a_{0 i}=0$ for every followeragent $i=1, \ldots, n$. For followers, we define a diagonal matrix $B=\operatorname{diag}\left\{a_{10}, \ldots, a_{n 0}\right\}$ to represent the leader-follower adjacency relationship. Let $H=L+B$.

Lemma 4 (see [11]). If vertex 0 is globally reachable in $\overrightarrow{\mathscr{G}}$, then all eigenvalues of $H$ have positive real-parts.

A Schur-complement lemma will be used in the stability analysis of the close-loop multi-agent systems and is given to end this subsection.

Lemma 5 (see [27]). Consider a symmetric matrix

$$
\Delta=\left(\begin{array}{ll}
\Delta_{11} & \Delta_{12} \\
\Delta_{12}^{T} & \Delta_{22}
\end{array}\right),
$$

where $\Delta_{11}$ and $\Delta_{22}$ are square. Then $\Delta$ is positive definite if and only if both $\Delta_{11}-\Delta_{12} \Delta_{22}^{-1} \Delta_{12}^{T}$ and $\Delta_{22}$ are positive definite.

2.2. Problem Formulation. In a leaderless consensus problem, a group of $n$ identical agents are moving with a continuoustime dynamics described by a second-order differential equation as follows:

$$
\begin{aligned}
& \dot{x}_{i}(t)=v_{i}(t), \\
& \dot{v}_{i}(t)=u_{i}(t), \quad i=1, \ldots, n,
\end{aligned}
$$

where $x_{i}(t) \in \mathbb{R}^{m}, v_{i}(t) \in \mathbb{R}^{m}$, and $u_{i}(t) \in \mathbb{R}^{m}$ are, respectively, the position, velocity, and control input of agent $i$.

In a leader-follower consensus problem, the dynamics of follower-agents are given as (3) while the kinematics of the self-active leader is described by the following second-order differential equation:

$$
\begin{aligned}
& \dot{x}_{0}(t)=v_{0}(t), \\
& \dot{v}_{0}(t)=a_{0}(t),
\end{aligned}
$$

where $x_{0}(t) \in \mathbb{R}^{m}, v_{0}(t) \in \mathbb{R}^{m}$, and $a_{0}(t) \in \mathbb{R}^{m}$ are, respectively, the position, velocity, and acceleration. Here for notation simplicity, let $m=1$.

When agents are equipped with resource-limited microprocessors, it is preferable to design an event-driven consensus controls for all agents such that the consensus controls need no update in continuous-time. For agent $i \in \mathscr{V}$, we define a state measurement error $e_{i}(t)$ and let $e(t)=$ $\operatorname{col}\left(e_{1}(t), \ldots, e_{n}(t)\right) \in \mathbb{R}^{n}$. Then an event-trigger condition is 
defined as $f(e(t), x(t), v(t))=0$, where $x(t), v(t) \in \mathbb{R}^{n}$ are, respectively, the concatenation vector of the state variables in system (3). All agents update their consensus controls $u_{i}(t)$ at a series of event-times $\tau(s)(s=0,1, \ldots)$ which are implicitly defined by $f(e(\tau(s)), x(\tau(s)), v(\tau(s)))=0$. Between control updates at two consecutive event-times, the eventdriven consensus control is held constant until the next event is triggered, that is,

$$
u_{i}(t)=u_{i}(\tau(s)), \quad \forall t \in[\tau(s), \tau(s+1)) .
$$

We say that the event-driven consensus problem of leaderless multi-agent system (3) is solved if an event-driven consensus control can be found to ensure that

$$
\begin{aligned}
& \lim _{t \rightarrow \infty}\left|x_{i}(t)-x_{j}(t)\right|=0, \\
& \lim _{t \rightarrow \infty}\left|v_{i}(t)-v_{j}(t)\right|=0,
\end{aligned}
$$

for $i, j \in \mathscr{V}$. Similarly, the event-driven consensus problem of leader-follower multi-agent system (3)-(4) is solved if

$$
\begin{aligned}
& \lim _{t \rightarrow \infty}\left|x_{i}(t)-x_{0}(t)\right|=0, \\
& \lim _{t \rightarrow \infty}\left|v_{i}(t)-v_{0}(t)\right|=0,
\end{aligned}
$$

for $i \in \mathscr{V}$.

\section{Control Design and Stability Analysis}

3.1. Leaderless Consensus Control. We assume that the consensus laws $u_{i}(t)$ in system (3) are updated only at discrete event-times. Suppose that the event-times of all agents are modeled as a sequence $\tau(s)$ for $s=0,1, \ldots$. For agent $i$ with dynamics (3), define two measurement errors $e_{i, x}(t)=$ $x_{i}(\tau(s))-x_{i}(t), e_{i, v}(t)=v_{i}(\tau(s))-v_{i}(t), t \in[\tau(s), \tau(s+1))$. The event-times $\tau(s)$ are defined by the function $f(e(\tau(s))$, $x(\tau(s)), v(\tau(s)))=0$, which will be determined in sequel.

An event-driven consensus control of agent $i$ is proposed as follows:

$$
\begin{aligned}
u_{i}(t)= & k^{2} \sum_{j \in \mathcal{N}_{i}} a_{i j}\left[x_{j}(\tau(s))-x_{i}(\tau(s))\right] \\
& +k^{3} \sum_{j \in \mathscr{N}_{i}} a_{i j}\left[v_{j}(\tau(s))-v_{i}(\tau(s))\right],
\end{aligned}
$$

for $t \in[\tau(s), \tau(s+1))$. The control gain parameter $k$ will be determined in sequel.

Then a compact form of (3) using control (8) is given by

$$
\begin{gathered}
\dot{x}(t)=v(t), \\
\dot{v}(t)=-k^{2} L x(\tau(s))-k^{3} L v(\tau(s)),
\end{gathered}
$$

where $x, v \in \mathbb{R}^{n}, L$ is the Laplacian matrix.

Denote $e_{x}(t)=\operatorname{col}\left(e_{1, x}(t), \ldots, e_{n, x}(t)\right), e_{v}(t)=\operatorname{col}\left(e_{1, v}(t)\right.$, $\left.\ldots, e_{n, v}(t)\right)$. One has $x(\tau(s))=e_{x}(t)+x(t), v(\tau(s))=e_{v}(t)+$ $v(t)$. Furthermore, system (9) can be rewritten as

$$
\begin{gathered}
\dot{x}(t)=v(t), \\
\dot{v}(t)=-k^{2} L x(t)-k^{3} L v(t)-k^{2} L e_{x}(t)-k^{3} L e_{v}(t),
\end{gathered}
$$

or

$$
\left(\begin{array}{l}
\dot{x}(t) \\
\dot{v}(t)
\end{array}\right)=F_{1}\left(\begin{array}{l}
x(t) \\
v(t)
\end{array}\right)+J_{1}\left(\begin{array}{l}
e_{x}(t) \\
e_{v}(t)
\end{array}\right),
$$

where

$$
F_{1}=\left(\begin{array}{cc}
0_{n \times n} & I_{n} \\
-k^{2} L & -k^{3} L
\end{array}\right), \quad J_{1}=\left(\begin{array}{cc}
0_{n \times n} & 0_{n \times n} \\
-k^{2} L & -k^{3} L
\end{array}\right) .
$$

Now we will analyze the convergence of the closed-loop multi-agent system (11) under the event-driven consensus control (8). To facilitate the convergence analysis, we describe the following lemma. Its proof is quite obvious and omitted here.

Lemma 6. For Laplacian matrix L associated with the directed graph $\mathscr{G}$, there exists a non-singular matrix

$$
U=\left(\begin{array}{cccc}
1 & * & \cdots & * \\
1 & * & \cdots & * \\
\vdots & \vdots & & \vdots \\
1 & * & \cdots & *
\end{array}\right) \in \mathbb{R}^{n \times n}
$$

such that

$$
U^{-1} L U=\left(\begin{array}{cc}
0 & h^{T} \\
0_{n-1} & \bar{H}
\end{array}\right)=\Lambda \in \mathbb{R}^{n \times n},
$$

where $h \in \mathbb{R}^{n-1}, \bar{H} \in \mathbb{R}^{(n-1) \times(n-1)}$.

From Lemma 6, the submatrix $\bar{H}$ of $\Lambda$ is a full-rank matrix if and only if $\mathscr{G}$ has a global reachable vertex. Moreover, the eigenvalues of $\bar{H}$ have positive real-parts, or equivalently, $-\bar{H}$ is Hurwitz stable. Therefore, there exists a positive definite matrix $\bar{P} \in \mathscr{R}^{(n-1) \times(n-1)}$ such that

$$
\bar{P} \bar{H}+\bar{H}^{T} \bar{P}=I_{n-1} .
$$

Take a coordinate transformation

$$
\begin{array}{ll}
\bar{x}(t)=U^{-1} x(t), & \bar{v}(t)=U^{-1} v(t), \\
\bar{e}_{x}(t)=U^{-1} e_{x}(t), & \bar{e}_{v}(t)=U^{-1} e_{v}(t),
\end{array}
$$

and then system (10) becomes

$$
\begin{gathered}
\dot{\bar{x}}(t)=\bar{v}(t), \\
\dot{\bar{v}}(t)=-k^{2} \Lambda \bar{x}(t)-k^{3} \Lambda \bar{v}(t)-k^{2} \Lambda \bar{e}_{x}(t)-k^{3} \Lambda \bar{e}_{v}(t) .
\end{gathered}
$$


Essentially, system (17) can be regarded as a series interconnection of two subsystems:

$$
\begin{aligned}
& \dot{\bar{x}}_{1}(t)=\bar{v}_{1}(t), \\
\dot{\bar{v}}_{1}(t)= & -k^{2} h^{T} \bar{x}_{2}(t)-k^{3} h^{T} \bar{v}_{2}(t) \\
& -k^{2} h^{T} \bar{e}_{x}^{2}(t)-k^{3} h^{T} \bar{e}_{v}^{2}(t), \\
& \dot{\bar{x}}_{2}(t)=\bar{v}_{2}(t), \\
\dot{\bar{v}}_{2}(t)= & -k^{2} \bar{H} \bar{x}_{2}(t)-k^{3} \bar{H} \bar{v}_{2}(t) \\
& -k^{2} \bar{H} \bar{e}_{x}^{2}(t)-k^{3} \bar{H} \bar{e}_{v}^{2}(t),
\end{aligned}
$$

where $\bar{x}_{1}, \bar{v}_{1}, \bar{e}_{x}^{1}, \bar{e}_{v}^{1} \in \mathbb{R}$ and $\bar{x}_{2}, \bar{v}_{2}, \bar{e}_{x}^{2}, \bar{e}_{v}^{2} \in \mathbb{R}^{n-1}$,

$$
\bar{x}=\left(\begin{array}{c}
\bar{x}_{1} \\
\bar{x}_{2}
\end{array}\right), \quad \bar{v}=\left(\begin{array}{c}
\bar{v}_{1} \\
\bar{v}_{2}
\end{array}\right), \quad \bar{e}_{x}=\left(\begin{array}{c}
\bar{e}_{x}^{1} \\
\bar{e}_{x}^{2}
\end{array}\right), \quad \bar{e}_{v}=\left(\begin{array}{c}
\bar{e}_{v}^{1} \\
\bar{e}_{v}^{2}
\end{array}\right) \text {. }
$$

Let $\epsilon=\operatorname{col}\left(\bar{x}_{2}, \bar{v}_{2}\right), e=\operatorname{col}\left(\bar{e}_{x}^{2}, \bar{e}_{v}^{2}\right)$, then subsystem (19) becomes

$$
\dot{\epsilon}=F_{2} \epsilon+J_{2} e,
$$

where

$$
\begin{aligned}
& F_{2}=\left(\begin{array}{cc}
0_{(n-1) \times(n-1)} & I_{n-1} \\
-k^{2} \bar{H} & -k^{3} \bar{H}
\end{array}\right), \\
& J_{2}=\left(\begin{array}{cc}
0_{(n-1) \times(n-1)} & 0_{(n-1) \times(n-1)} \\
-k^{2} \bar{H} & -k^{3} \bar{H}
\end{array}\right) .
\end{aligned}
$$

Now a main result is obtained for system (10).

Theorem 7. Assume that the interconnection topology $\mathscr{G}$ associated with multi-agent system (3) has a globally reachable vertex. If the control gain $k$ satisfies

$$
k>\sqrt{\frac{\lambda_{\max }(\bar{P})}{2}+1}
$$

with $\bar{P}$ given in (15), then the event-driven consensus problem of the leaderless multi-agent system (3) is solved with the control (8), that is,

$$
\begin{aligned}
& \lim _{t \rightarrow \infty}\left\|x_{i}(t)-x_{j}(t)\right\|=0, \\
& \lim _{t \rightarrow \infty}\left\|v_{i}(t)-v_{j}(t)\right\|=0 .
\end{aligned}
$$

Proof. For system (19), or equivalently (21), take a Lyapunov function

$$
V(\epsilon)=\epsilon^{T} P \epsilon,
$$

where

$$
P=\left(\begin{array}{ll}
k & 1 \\
1 & k
\end{array}\right) \otimes \bar{P}
$$

where $\bar{P}$ satisfies (15). Thus, $P$ is positive definite with $k$ given in (23).
Differentiating $V(\epsilon)$ leads to

$$
\begin{aligned}
\left.\dot{V}(\epsilon)\right|_{(21)} & =\epsilon^{T}\left(F_{2}^{T} P+P F_{2}\right) \epsilon+2 \epsilon^{T} P J_{2} \mathrm{e} \\
& =-\epsilon^{T} Q \epsilon+2 \epsilon^{T} P J_{2} \mathrm{e},
\end{aligned}
$$

where

$$
\begin{aligned}
Q & =-\left(F_{2}^{T} P+P F_{2}\right) \\
& =\left(\begin{array}{cc}
k^{2} I_{n-1} & k^{3} I_{n-1}-k \bar{P} \\
k^{3} I_{n-1}-k \bar{P} & k^{4} I_{n-1}-2 \bar{P}
\end{array}\right) .
\end{aligned}
$$

According to Schur complement Lemma 5, Q is positive definite with $k$ given in (23).

Therefore,

$$
\begin{aligned}
\left.\dot{V}(\epsilon)\right|_{(21)} & \leq-\lambda_{\min }(Q)\|\epsilon\|^{2}+2 \lambda_{\max }(P)\left\|J_{2}\right\|\|\epsilon\|\|e\| \\
& =-\|\epsilon\|\left[\lambda_{\min }(Q)\|\epsilon\|-2 \lambda_{\max }(P)\left\|J_{2}\right\|\|e\|\right] .
\end{aligned}
$$

Due to the fact that $\left\|J_{2}\right\|^{2}=k^{4}\left(k^{2}+1\right)\|\bar{H}\|^{2}$, enforcing the measurement error $e$ to satisfy

$$
\|e\| \leq \sigma \frac{\lambda_{\min }(Q)\|\epsilon\|}{2 k^{2} \sqrt{k^{2}+1} \lambda_{\max }(P)\|\bar{H}\|}
$$

with $\sigma \in(0,1)$, one has

$$
\begin{aligned}
\dot{V}(\epsilon) & \leq-(1-\sigma) \lambda_{\min }(Q)\|\epsilon\|^{2} \\
& \leq-\frac{(1-\sigma) \lambda_{\min }(Q)}{\lambda_{\max }(P)} V(\epsilon) .
\end{aligned}
$$

From (31), we conclude that $\lim _{t \rightarrow \infty} \epsilon(t)=0$, that is, as $t \rightarrow$ $\infty, \bar{x}_{2}(t) \rightarrow 0, \bar{v}_{2}(t) \rightarrow 0$.

On the other hand, for system (18), let $\bar{x}_{1}(0), \bar{v}_{1}(0)$ be the initial values of $\bar{x}_{1}(t), \bar{v}_{1}(t)$ and take a variable change $\widetilde{x}_{1}(t)=$ $\bar{x}_{1}(t)-\left(\bar{v}_{1}(0) t+\bar{x}_{1}(0)\right), \widetilde{v}_{1}(t)=\bar{v}_{1}(t)-\bar{v}_{1}(0)$. Then the solution can be described by the following integral equation:

$$
\begin{aligned}
& \left(\begin{array}{l}
\widetilde{x}_{1} \\
\widetilde{v}_{1}
\end{array}\right)=\int_{0}^{t}\left(\begin{array}{cc}
1 & t-\varsigma \\
0 & 1
\end{array}\right) \\
& \times\left(-k^{2} h^{T} \bar{x}_{2}(\varsigma)-k^{3} h^{T} \bar{v}_{2}(\varsigma)-k^{2} h^{T} \bar{e}_{x}^{2}(\varsigma)-k^{3} h^{T} \bar{e}_{v}^{2}(\varsigma)\right) d \varsigma .
\end{aligned}
$$

Since the system (19) is exponentially stable, based on the event-triggered condition (30), solution (32) has an exponential decay term with respect to time $t$. Consequently, the solution is convergent to 0 as $t \rightarrow \infty$, and thus,

$$
\begin{gathered}
\left(\begin{array}{c}
\bar{x}_{1}(t) \\
\bar{x}_{2}(t)
\end{array}\right)-\left(\begin{array}{c}
\bar{v}_{1}(0) t+\bar{x}_{1}(0) \\
0_{n-1}
\end{array}\right) \longrightarrow 0_{n}, \\
\left(\begin{array}{c}
\bar{v}_{1}(t) \\
\bar{v}_{2}(t)
\end{array}\right) \longrightarrow\left(\begin{array}{c}
\bar{v}_{1}(0) \\
0_{n-1}
\end{array}\right),
\end{gathered}
$$


as $t \rightarrow \infty$. Furthermore, from the variable change (16), one has

$$
\begin{aligned}
x & -U\left(\begin{array}{c}
\bar{v}_{1}(0) t+\bar{x}_{1}(0) \\
0_{n-1}
\end{array}\right) \\
& =x-\mathbf{1}_{n} \otimes\left(\bar{v}_{1}(0) t+\bar{x}_{1}(0)\right) \longrightarrow 0_{n}, \\
v & -U\left(\begin{array}{c}
\bar{v}_{1}(0) \\
0_{n-1}
\end{array}\right)=v-\mathbf{1}_{n} \otimes \bar{v}_{1}(0) \longrightarrow 0_{n} .
\end{aligned}
$$

Therefore, when the event-driven consensus control (8) is applied to each agent, one has $x_{i}(t)-x_{j}(t) \rightarrow 0, v_{i}(t)-$ $v_{j}(t) \rightarrow 0$ as $t \rightarrow \infty$. The proof is complete.

Remark 8. The gain $k$ in (23) can be taken without exact knowledge of the interconnection topology associated with the multi-agent system in real application of the control (8). In fact, the spectral norm of $\bar{P}$ in (23) can be obtained by estimating the bound of the solution of Lyapunov equation (15) (see [28]), which is closely related with the Laplacian spectrum. Fortunately, there have been many results on the bounds of the eigenvalues of a Laplacian matrix [29].

Next, we show that the inter-event times $\tau(\mathrm{s}+1)-\tau(s)$ have a lower bound for $s=0,1, \ldots$ It is noticed that, from (16),

$$
\begin{aligned}
& \left(\begin{array}{l}
\bar{x}(t) \\
\bar{v}(t)
\end{array}\right)=\left(I_{2} \otimes U^{-1}\right)\left(\begin{array}{l}
x(t) \\
v(t)
\end{array}\right), \\
& \left(\begin{array}{l}
\bar{e}_{x}(t) \\
\bar{e}_{v}(t)
\end{array}\right)=\left(I_{2} \otimes U^{-1}\right)\left(\begin{array}{l}
e_{x}(t) \\
e_{v}(t)
\end{array}\right) .
\end{aligned}
$$

Define a matrix $W=\left[0_{n-1} I_{n-1}\right] \in \mathbb{R}^{(n-1) \times n}$. Then, based on (35), one has

$$
\begin{gathered}
\epsilon=\left(I_{2} \otimes W\right)\left(\begin{array}{l}
\bar{x}(t) \\
\bar{v}(t)
\end{array}\right)=\left(I_{2} \otimes W U^{-1}\right)\left(\begin{array}{l}
x(t) \\
v(t)
\end{array}\right), \\
e=\left(I_{2} \otimes W U^{-1}\right)\left(\begin{array}{c}
e_{x}(t) \\
e_{v}(t)
\end{array}\right) .
\end{gathered}
$$

For convenience of simplified calculation, let $p(t)=\operatorname{col}(x(t)$, $v(t)), e_{p}(t)=\operatorname{col}\left(e_{x}(t), e_{v}(t)\right)$ and $z(t)=\left\|e_{p}(t)\right\| /\|p(t)\|$. Take the derivative of $z(t)$ :

$$
\begin{aligned}
\dot{z} & =\frac{d}{d t} \frac{\left(e_{p}^{T} e_{p}\right)^{1 / 2}}{\left(p^{T} p\right)^{1 / 2}}=-\frac{e_{p}^{T} \dot{p}}{\left\|e_{p}\right\|\|p\|}-\frac{\left\|e_{p}\right\|}{\|p\|} \frac{p^{T} \dot{p}}{\|p\|^{2}} \\
& \leq\left[1+\frac{\left\|e_{p}\right\|}{\|p\|}\right] \frac{\|\dot{p}\|}{\|p\|}=(1+z)\left(\left\|F_{1}\right\|+\left\|J_{1}\right\| z\right) .
\end{aligned}
$$

Assume that $\phi\left(t, \phi_{0}\right)$ is the solution of

$$
\dot{\phi}=(1+\phi)\left(\left\|F_{1}\right\|+\left\|J_{1}\right\| \phi\right), \phi\left(0, \phi_{0}\right)=\phi_{0} .
$$

Then, one has $z(t) \leq \phi\left(t, \phi_{0}\right)$ for $t \geq 0$. From the variable change (36) and the event-driven condition (30), $z(t)$ satisfies

$$
z(t)=\frac{\left\|e_{p}(t)\right\|}{\|p(t)\|}=\frac{\|e(t)\|}{\|\epsilon(t)\|}
$$

and the inter-event times are bounded by the time $\tau_{D} \in \mathbb{R}^{+}$ it takes for $\phi$ to evolve from 0 to $\phi\left(\tau_{D}, 0\right)=\left(\sigma \lambda_{\min }(Q) /\right.$ $\left.2 k^{2} \sqrt{k^{2}+1} \lambda_{\max }(P)\|\bar{H}\|\right)$. Solving the differential equation (38) leads to

$$
\tau_{D}=\frac{1}{\left\|F_{1}\right\|-\left\|J_{1}\right\|} \ln \left(\frac{1+\phi\left(\tau_{D}, 0\right)}{1+\left\|J_{1}\right\| /\left\|F_{1}\right\| \phi\left(\tau_{D}, 0\right)}\right),
$$

which is a positive bound due to the fact that $\left\|F_{1}\right\|>\left\|J_{1}\right\|$.

Then a result is stated as follows.

Theorem 9. When the event-driven consensus control (8) is applied to the multi-agent system (3), the inter-event times $\tau(s+$ $1)-\tau(s)$ for $s=0,1, \ldots$, have a lower bound given by (40).

3.2. Leader-Follower Consensus Control. In the leaderfollowing problem, we assume that the state information, that is, $x_{0}(t), v_{0}(t)$, and $a_{0}(t)$ of the leader can be measured in continuous-time by the followers. Thus, we propose the following event-driven consensus control of the follower $i$ :

$$
\begin{aligned}
u_{i}(t)= & a_{0}(t)+k^{2} \sum_{j \in \mathcal{N}_{i}} a_{i j}\left[x_{j}(\tau(s))-x_{i}(\tau(s))\right] \\
& +k^{2} a_{i 0}\left[x_{0}(t)-x_{i}(t)\right] \\
& +k^{3} \sum_{j \in \mathcal{N}_{i}} a_{i j}\left[v_{j}(\tau(s))-v_{i}(\tau(s))\right] \\
& +k^{3} a_{i 0}\left[v_{0}(t)-v_{i}(t)\right]
\end{aligned}
$$

for $t \in[\tau(s), \tau(s+1))$.

Remark 10. For the leader-following problem (3)-(4) under investigation, the leader is assumed to be self-active, which means that the leader is moving according to its own (predesigned) policy and needs no feedback information from any other agent. In some sense, the leader plays the role of an external commander of all the followers. Thus, no eventdriven strategy is applied to update the state of the leader. Though the followers are using the event-driven relative information from the neighboring followers, but they can obtain the real-time relative position and velocity measurements from the leader only if the followers are connected to the leader. Therefore, in the control (41), it would be preferable to assume that $x_{0}(t)-x_{i}(t)$ and $v_{0}(t)-v_{i}(t)$ are measured in continuous time.

Still denote the measurement errors $e_{i, x}(t)=x_{i}(\tau(s))-$ $x_{i}(t), e_{i, v}(t)=v_{i}(\tau(s))-v_{i}(t)$ for $i=1, \ldots, n, t \in[\tau(s), \tau(s+1))$. Applying control (41) to system (3) leads to

$$
\begin{aligned}
\dot{x}(t)= & v(t) \\
\dot{v}(t)= & a_{0}(t) \mathbf{1}-k^{2} H x(t)+k^{2} B \mathbf{1} x_{0}(t) \\
& -k^{3} H v(t)+k^{3} B \mathbf{1} v_{0}(t) \\
& -k^{2} L e_{x}(t)-k^{3} L e_{v}(t)
\end{aligned}
$$


where $H=L+B$. Define $\widetilde{x}_{i}(t)=x_{i}(t)-x_{0}(t), \widetilde{v}_{i}(t)=$ $v_{i}(t)-v_{0}(t)$ and $\widetilde{x}(t)=\operatorname{col}\left(\widetilde{x}_{1}(t), \ldots, \widetilde{x}_{n}(\mathrm{t})\right), \widetilde{v}(t)=\operatorname{col}\left(\widetilde{v}_{1}(t)\right.$, $\left.\ldots, \widetilde{v}_{n}(t)\right)$. Based on the fact that $-H \tilde{x}(t)=-H x(t)+B \mathbf{1} x_{0}(t)$, $-H \widetilde{v}(t)=-H v(t)+B \mathbf{1} v_{0}(t)$, one has

$$
\left(\begin{array}{c}
\dot{\tilde{x}}(t) \\
\dot{\tilde{v}}(t)
\end{array}\right)=F_{3}\left(\begin{array}{c}
\tilde{x}(t) \\
\widetilde{v}(t)
\end{array}\right)+J_{3}\left(\begin{array}{c}
e_{x}(t) \\
e_{v}(t)
\end{array}\right),
$$

where

$$
F_{3}=\left(\begin{array}{cc}
0_{n \times n} & I_{n} \\
-k^{2} H & -k^{3} H
\end{array}\right), \quad J_{3}=\left(\begin{array}{cc}
0_{n \times n} & 0_{n \times n} \\
-k^{2} L & -k^{3} L
\end{array}\right) .
$$

Theorem 11. Assume that the vertex 0 is globally reachable in the leader-follower interconnection topology $\vec{G}$. If the control gain $k$ satisfies

$$
k>\sqrt{\frac{\lambda_{\max }(\widetilde{P})}{2}+1},
$$

where $\widetilde{P}$ is the solution of the Lyapunov equation $\widetilde{P} H+H^{T} \widetilde{P}=$ $I_{n}$, then the event-driven consensus problem of the leaderfollower multi-agent system (3)-(4) is solved with the control (41), that is,

$$
\begin{aligned}
& \lim _{t \rightarrow \infty}\left\|x_{i}(t)-x_{0}(t)\right\|=0, \\
& \lim _{t \rightarrow \infty}\left\|v_{i}(t)-v_{0}(t)\right\|=0 .
\end{aligned}
$$

Proof. For system (43), take a Lyapunov function

$$
V(\varepsilon)=\varepsilon^{T} P_{L} \varepsilon,
$$

where

$$
\varepsilon(t)=\left(\begin{array}{l}
\tilde{x}(t) \\
\widetilde{v}(t)
\end{array}\right), \quad P_{L}=\left(\begin{array}{cc}
k & 1 \\
1 & k
\end{array}\right) \otimes \widetilde{P} .
$$

It is not difficult to show that $P_{L}$ is positive definite with $k$ given in (45).

Differentiating $V(\varepsilon)$ leads to

$$
\begin{aligned}
\left.\dot{V}(\varepsilon)\right|_{(43)} & =\varepsilon^{T}\left(F_{3}^{T} P_{L}+P_{L} F_{3}\right) \varepsilon+2 \varepsilon^{T} P_{L} J_{3} e \\
& =-\varepsilon^{T} Q_{L} \varepsilon+2 \varepsilon^{T} P_{L} J_{3} e,
\end{aligned}
$$

where

$$
Q_{L}=-\left(F_{3}^{T} P_{L}+P_{L} F_{3}\right)=\left(\begin{array}{cc}
k^{2} I_{n} & k^{3} I_{n}-k \widetilde{P} \\
k^{3} I_{n}-k \widetilde{P} & k^{4} I_{n}-2 \widetilde{P}
\end{array}\right) .
$$

According to Schur complement Lemma 5, $Q_{L}$ is positive definite with $k$ given in (45).

Therefore,

$$
\begin{aligned}
\left.\dot{V}(\varepsilon)\right|_{(43)} & \leq-\lambda_{\min }\left(Q_{L}\right)\|\epsilon\|^{2}+2 \lambda_{\max }\left(P_{L}\right)\left\|J_{3}\right\|\|\varepsilon\|\|e\| \\
& =-\|\varepsilon\|\left[\lambda_{\min }\left(Q_{L}\right)\|\epsilon\|-2 \lambda_{\max }\left(P_{L}\right)\left\|J_{3}\right\|\|e\|\right] .
\end{aligned}
$$

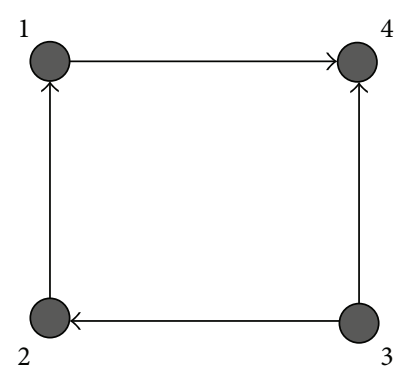

FIGURE 1: The interconnection topology $\mathscr{G}$.

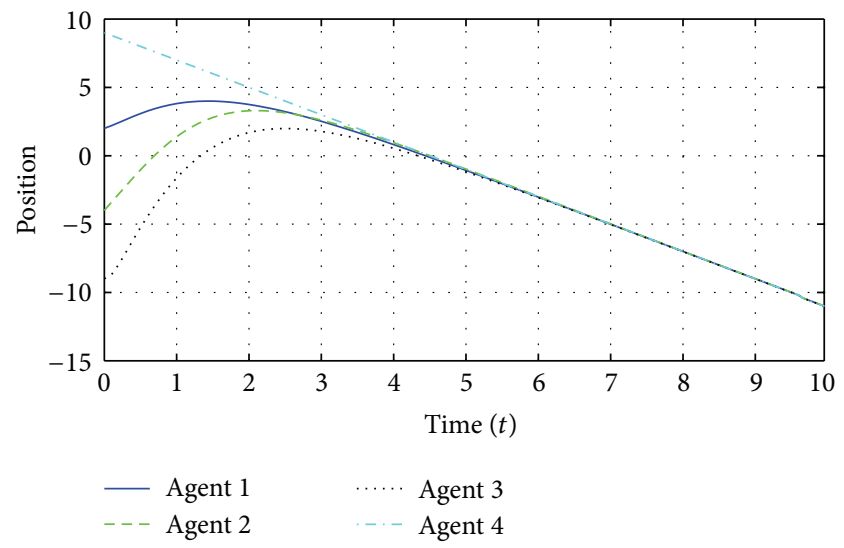

Figure 2: The evolution of position states $x_{i}(t)$ for $i=1, \ldots, 4$.

Due to the fact that $\left\|J_{3}\right\|^{2}=k^{4}\left(k^{2}+1\right)\|H\|^{2}$, enforcing the measurement error $e(t)$ to satisfy

$$
\|e(t)\| \leq \sigma \frac{\lambda_{\min }\left(Q_{L}\right)\|\varepsilon\|}{2 k^{2} \sqrt{k^{2}+1} \lambda_{\max }\left(P_{L}\right)\|\bar{H}\|}
$$

with $\sigma \in(0,1)$, one has

$$
\begin{aligned}
\dot{V}(\varepsilon) & \leq-(1-\sigma) \lambda_{\min }\left(Q_{L}\right)\|\varepsilon\|^{2} \\
& \leq-\frac{(1-\sigma) \lambda_{\min }\left(Q_{L}\right)}{\lambda_{\max }\left(P_{L}\right)} V(\varepsilon) .
\end{aligned}
$$

From (53), we conclude that $\lim _{t \rightarrow \infty} \varepsilon(t)=0$, that is, as $t \rightarrow$ $\infty, \widetilde{x}_{2}(t) \rightarrow 0, \widetilde{v}_{2}(t) \rightarrow 0$. The proof is thus competed.

Similar to Theorem 9, a conclusion about the lower bound of the event-times can also be found true, which is omitted here.

\section{Simulations}

Example 12. Consider four agents whose dynamics is described by (3). The interconnection topology is shown in Figure 1. Obviously, $\mathscr{G}$ has a globally vertex 4 ; however, it is not strongly connected. 


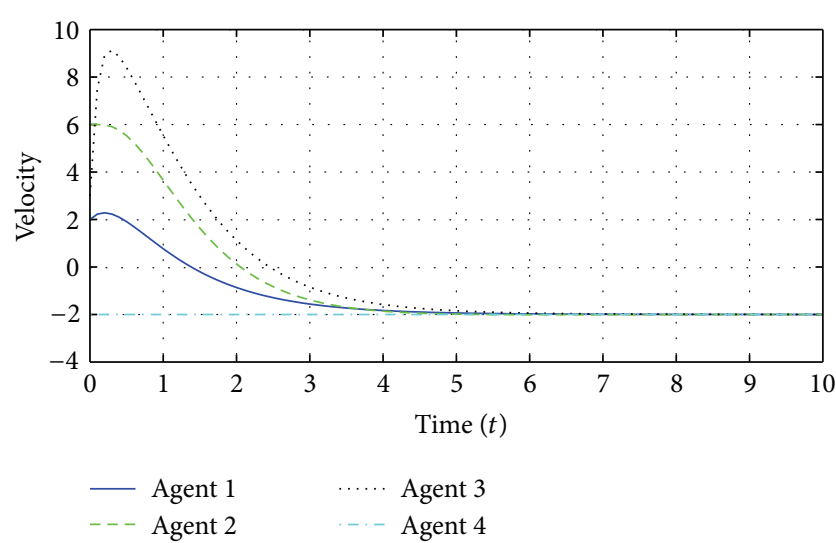

FIGURE 3: The evolution of velocity state $v_{i}(t)$ for $i=1, \ldots, 4$.

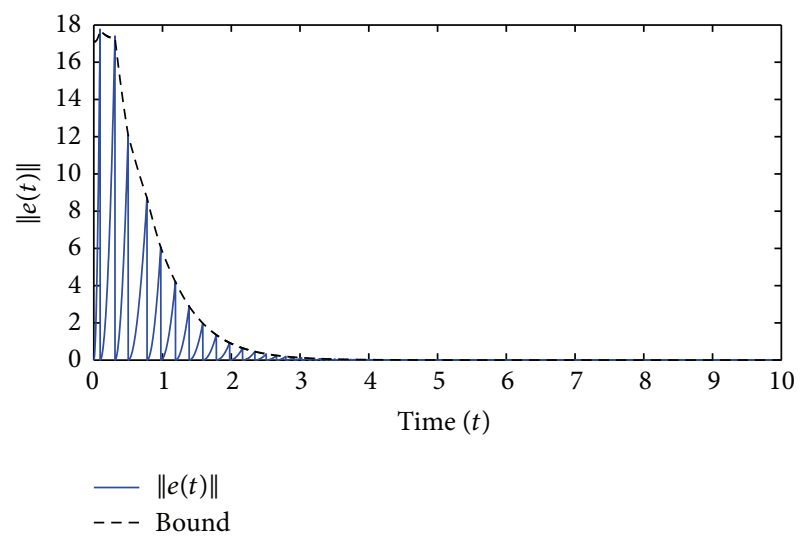

FIGURE 4: Evolution of the measurement error norm $\|e(t)\|$.

Assume that the weighted adjacency matrix $A$ reduces to a $0-1$ matrix. Then the Laplacian matrix $L$ of $\mathscr{G}$ is

$$
L=\left(\begin{array}{cccc}
1 & 0 & 0 & -1 \\
-1 & 1 & 0 & 0 \\
0 & -1 & 2 & -1 \\
0 & 0 & 0 & 0
\end{array}\right)
$$

which is an asymmetric matrix. A non-singular matrix $U$ in Lemma 6 can be easily found as

$$
U=\left(\begin{array}{llll}
1 & 0 & 0 & 0 \\
1 & 1 & 0 & 0 \\
1 & 0 & 1 & 0 \\
1 & 0 & 0 & 1
\end{array}\right) .
$$

Then the submatrices $\bar{H}=\left(\begin{array}{ccc}1 & 0 & 1 \\ -1 & 2 & 0 \\ 0 & 0 & 1\end{array}\right)$ and $h=\operatorname{col}(0,0,-1)$. The positive definite matrix satisfying (15) is given by

$$
\bar{P}=\left(\begin{array}{ccc}
0.5833 & 0.0833 & -0.3056 \\
0.0833 & 0.2500 & -0.0278 \\
-0.3056 & -0.0278 & 0.8056
\end{array}\right)
$$

whose minimal and maximal eigenvalues are, respectively, $\lambda_{\text {min }}(\bar{P})=0.2248, \lambda_{\max }(\bar{P})=1.0260$. According to (23), take the gain $k=1.5$.

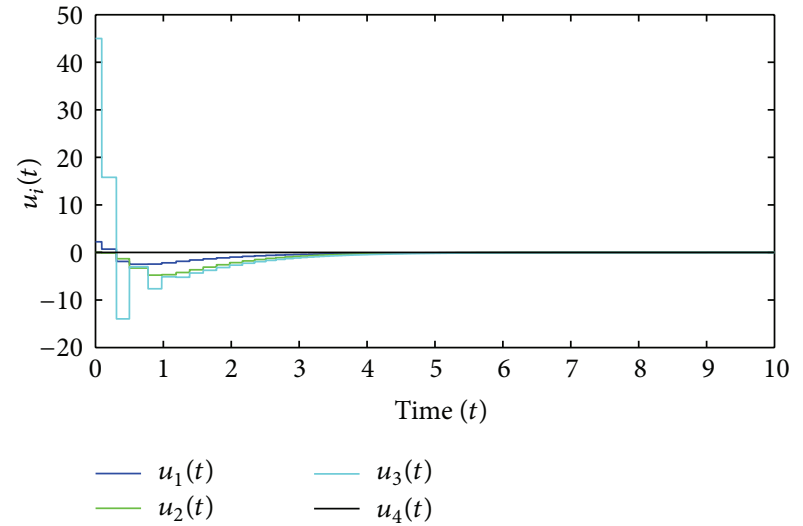

FIGURE 5: Evolution of the consensus controllers $u_{i}(t)$ for $i=$ $1, \ldots, 4$.

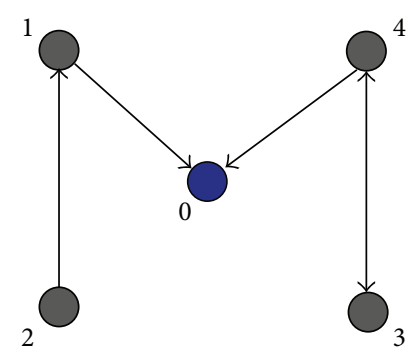

FIGURE 6: $\overrightarrow{\mathscr{V}}(\overrightarrow{\mathscr{G}})=\{0,1,2,3,4\}$ and $\mathscr{V}(\mathscr{G})=\{1,2,3,4\}$.

The initial conditions of system (9) are $x(0)=\operatorname{col}(2,-4$, $-9,9), v(0)=\operatorname{col}(2,6,3,-2)$ and $\tau(0)=0$. In the event triggered condition (30), we take $\sigma=0.8$. It can be seen that, from Figures 2 and 3, the four agents reach consensus on the position and velocity states with the proposed eventdriven consensus control (8). Additionally, since the vertex 4 has no link starting from it, the agent 4 is moved according to its own dynamics and the initial conditions, as shown in Figures 2, 3, and 5. The evolution of the measurement error vector $e(t)$ is depicted in Figure 4, which shows that the error $e(t)$ is bounded by the specified threshold $\left(\sigma \lambda_{\min }(Q)\|\epsilon\| / 2 k^{2} \sqrt{k^{2}+1} \lambda_{\max }(P)\|\bar{H}\|\right)$. In Figure 5 , the consensus controllers are illustrated for the four agents, whose event-driven update frequencies are decreasing as time evolves.

Example 13. Consider four followers and one leader whose dynamics are, respectively, described by (3) and (4). The leader-follower interconnection topology $\overrightarrow{\mathscr{G}}$ is shown in Figure 6, which has a globally reachable vertex 0 .

It is not difficult to obtain the Laplacian matrix $L$ as

$$
L=\left(\begin{array}{cccc}
1 & -1 & 0 & 0 \\
-1 & 1 & 0 & 0 \\
0 & 0 & 1 & -1 \\
0 & 0 & -1 & 1
\end{array}\right)
$$

and the leader adjacency matrix $B=\operatorname{diag}\{1,0,0,1\}$. Since $-H=-(L+B)$ is a stable matrix, one solves the Lyapunov 


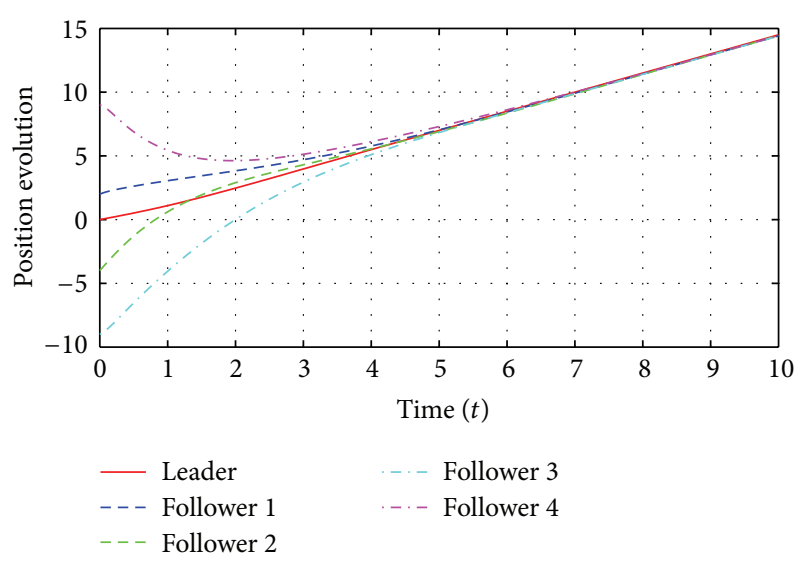

FIGURE 7: The evolution of position states $x_{i}(t)$ for $i=0,1, \ldots, 4$.

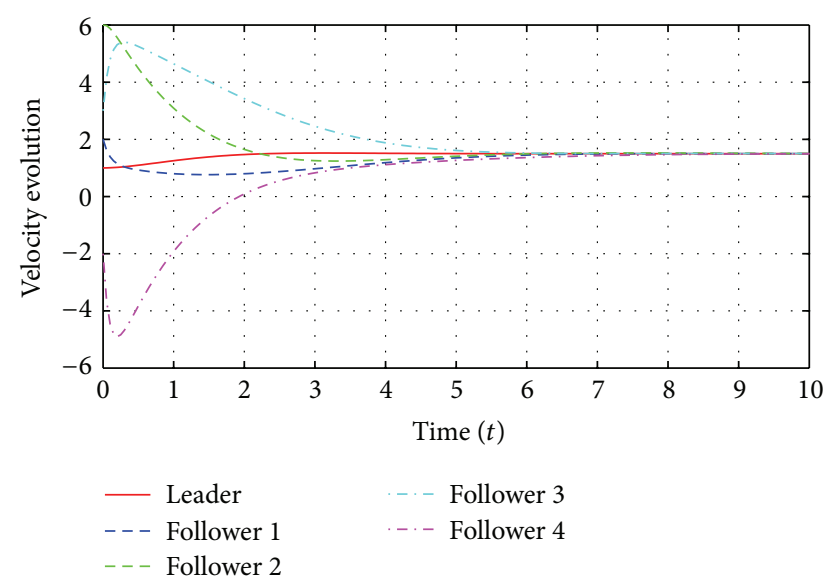

FIGURE 8: The evolution of velocity states $v_{i}(t)$ for $i=0,1, \ldots, 4$.

equation $\widetilde{P} H+H^{T} \widetilde{P}=I_{4}$ and has the positive definite solution as

$$
\widetilde{P}=\left(\begin{array}{cccc}
0.75 & 0.25 & 0 & 0 \\
0.25 & 0.50 & 0 & 0 \\
0 & 0 & 1.0 & 0.50 \\
0 & 0 & 0.50 & 0.50
\end{array}\right)
$$

whose maximal eigenvalue $\lambda_{\max }(H)=1.309$. From (45), still take $k=1.5$.

The acceleration of the active leader is assumed to be $a_{0}(t)=e^{-t} \sin (t)$. The initial values of system (3) is same as those in Example 12 and the initial values of the leader is given as $x_{0}(0)=0, v_{0}(0)=1$ and $a_{0}(0)=0$. Figures 7 and 8 show that the followers and the self-active leader reach consensus on the position and velocity under the event-driven control (41). The evolution of $\|e(t)\|$ is also presented in Figure 9.

\section{Conclusions}

An event-driven consensus problem of second-order multiagent systems with/without a self-active leader was considered in this paper. The consensus controllers have been proposed for all autonomous mobile agents based on an eventdriven control update strategy. The input-to-state stability

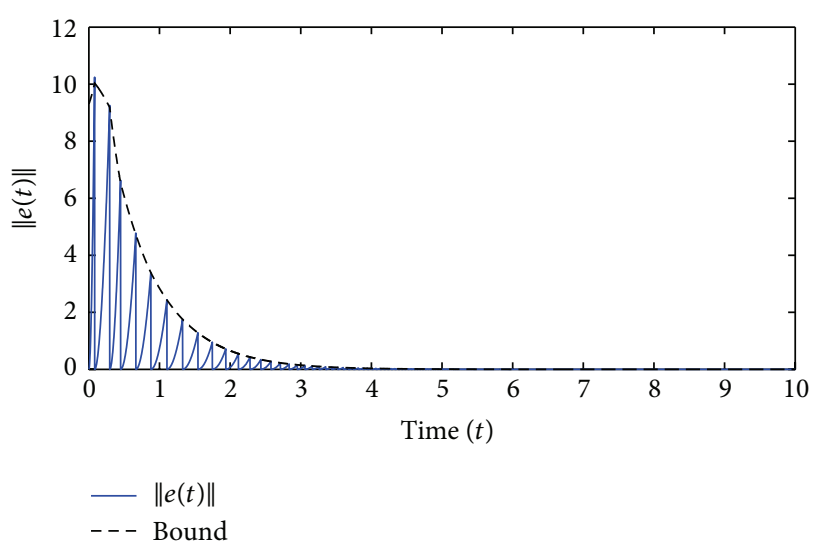

Figure 9: Evolution of the measurement error norm $\|e(t)\|$.

of the closed-loop multi-agent system has been analyzed by employing an ISS Lyapunov function. Some numerical examples have been presented to validate the proposed eventdriven controls. However, it is noted that the event-driven condition depends on the states of the whole multi-agent group and all agents have identical event-times. The result is somewhat preliminary due to the centralized information gathering, so further work will be devoted to designing a decentralized event-driven consensus control for a secondorder multi-agent system in the future.

\section{Acknowledgments}

This work is supported by the National Natural Science Foundation of China under Grant 61104104 and the Scientific Research Foundation for the Returned Overseas Chinese Scholars, State Education Ministry of China.

\section{References}

[1] M. H. DeGroot, "Reaching a consensus," Journal of the American Statistical Association, vol. 69, no. 345, pp. 118-121, 1974.

[2] R. Olfati-Saber, J. A. Fax, and R. M. Murray, "Consensus and cooperation in networked multi-agent systems," Proceedings of the IEEE, vol. 95, no. 1, pp. 215-233, 2007.

[3] A. Jadbabaie, J. Lin, and A. S. Morse, "Coordination of groups of mobile autonomous agents using nearest neighbor rules," IEEE Transactions on Automatic Control, vol. 48, no. 6, pp. 988-1001, 2003.

[4] R. Olfati-Saber and R. M. Murray, "Consensus problems in networks of agents with switching topology and time-delays," IEEE Transactions on Automatic Control, vol. 49, no. 9, pp. 15201533, 2004.

[5] J. Hu and G. Feng, "Distributed tracking control of leaderfollower multi-agent systems under noisy measurement," Automatica, vol. 46, no. 8, pp. 1382-1387, 2010.

[6] L. Moreau, "Stability of multiagent systems with time-dependent communication links," IEEE Transactions on Automatic Control, vol. 50, no. 2, pp. 169-182, 2005.

[7] J. Lorenz and D. A. Lorenz, "On conditions for convergence to consensus," IEEE Transactions on Automatic Control, vol. 55, no. 7, pp. 1651-1656, 2010. 
[8] Z.-J. Tang, T.-Z. Huang, and J.-L. Shao, "Containment control of multiagent systems with multiple leaders and noisy measurements," Abstract and Applied Analysis, vol. 2012, Article ID 262153, 9 pages, 2012.

[9] P. Lin, K. Qin, Z. Li, and W. Ren, "Collective rotating motions of second-order multi-agent systems in three-dimensional space," Systems \& Control Letters, vol. 60, no. 6, pp. 365-372, 2011.

[10] K. D. Do, "Formation control of multiple elliptical agents with limited sensing ranges," Automatica, vol. 48, no. 7, pp. 13301338, 2012.

[11] J. Hu and Y. Hong, "Leader-following coordination of multiagent systems with coupling time delays," Physica A, vol. 374, no. 2, pp. 853-863, 2007.

[12] Y. Cao, D. Stuart, W. Ren, and Z. Meng, "Distributed containment control for multiple autonomous vehicles with doubleintegrator dynamics: algorithms and experiments," IEEE Transactions on Control Systems Technology, vol. 19, no. 4, pp. 929938, 2011.

[13] Y. Hong, G. Chen, and L. Bushnell, "Distributed observers design for leader-following control of multi-agent networks," Automatica, vol. 44, no. 3, pp. 846-850, 2008.

[14] Z. Wu, L. Peng, L. Xie, and J. Wen, "Stochastic bounded consensus tracking of second-order multi-agent systems with measurement noises and sampled-data," Journal of Intelligent \& Robotic Systems, vol. 68, no. 3-4, pp. 261-273, 2012.

[15] J. Wang, D. Cheng, and X. Hu, "Consensus of multi-agent linear dynamic systems," Asian Journal of Control, vol. 10, no. 2, pp. 144-155, 2008.

[16] Y. Tian, "High-order consensus of heterogeneous multi-agent systems," in Proceedings of the 8th Asian Control Conference, pp. 341-346, Kaohsiung, Taiwan, 2011.

[17] X. Wang, Y. Hong, J. Huang, and Z.-P. Jiang, "A distributed control approach to a robust output regulation problem for multiagent linear systems," IEEE Transactions on Automatic Control, vol. 55, no. 12, pp. 2891-2895, 2010.

[18] J. Hu and G. Feng, "Quantized tracking control for a multiagent system with high-order leader dynamics," Asian Journal of Control, vol. 13, no. 6, pp. 988-997, 2011.

[19] Z. Li, Z. Duan, and G. Chen, "Dynamic consensus of linear multi-agent systems," IET Control Theory \& Applications, vol. 5, no. 1, pp. 19-28, 2011.

[20] P. Tabuada, "Event-triggered real-time scheduling of stabilizing control tasks," IEEE Transactions on Automatic Control, vol. 52, no. 9, pp. 1680-1685, 2007.

[21] Y. Gao and L. Wang, "Sampled-data based consensus of continuous-time multi-agent systems with time-varying topology," IEEE Transactions on Automatic Control, vol. 56, no. 5, pp. 12261231, 2011.

[22] Z.-J. Tang, T.-Z. Huang, J.-L. Shao, and J.-P. Hu, "Leaderfollowing consensus for multi-agent systems via sampled-data control," IET Control Theory \& Applications, vol. 5, no. 14, pp. 1658-1665, 2011.

[23] D. V. Dimarogonas, E. Frazzoli, and K. H. Johansson, "Distributed event-triggered control for multi-agent systems," IEEE Transactions on Automatic Control, vol. 57, no. 5, pp. 1291-1297, 2012.

[24] Z. Liu, Z. Chen, and Z. Yuan, "Event-triggered average-consensus of multi-agent systems with weighted and direct topology," Journal of Systems Science \& Complexity, vol. 25, no. 5, pp. 845-855, 2012.
[25] J. Hu, G. Chen, and H.-X. Li, "Distributed event-triggered tracking control of leader-follower multi-agent systems with communication delays," Kybernetika, vol. 47, no. 4, pp. 630-643, 2011.

[26] C. Godsil and G. Royle, Algebraic Graph Theory, vol. 207 of Graduate Texts in Mathematics, Springer, New York, NY, USA, 2001.

[27] R. A. Horn and C. R. Johnson, Matrix Analysis, Cambridge University Press, Cambridge, UK, 1985.

[28] T. Mori and I. A. Derese, "A brief summary of the bounds on the solution of the algebraic matrix equations in control theory," International Journal of Control, vol. 39, no. 2, pp. 247-256, 1984.

[29] R. Merris, "Laplacian matrices of graphs: a survey," Linear Algebra and Its Applications, vol. 197-198, pp. 143-176, 1994. 


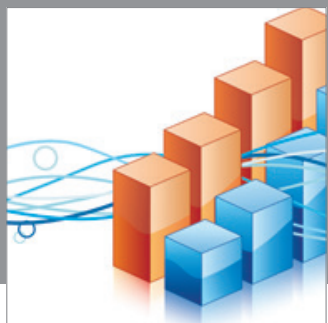

Advances in

Operations Research

mansans

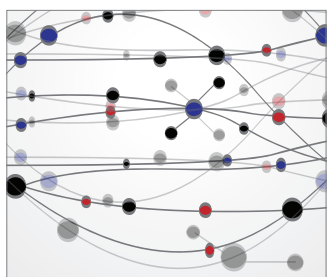

The Scientific World Journal
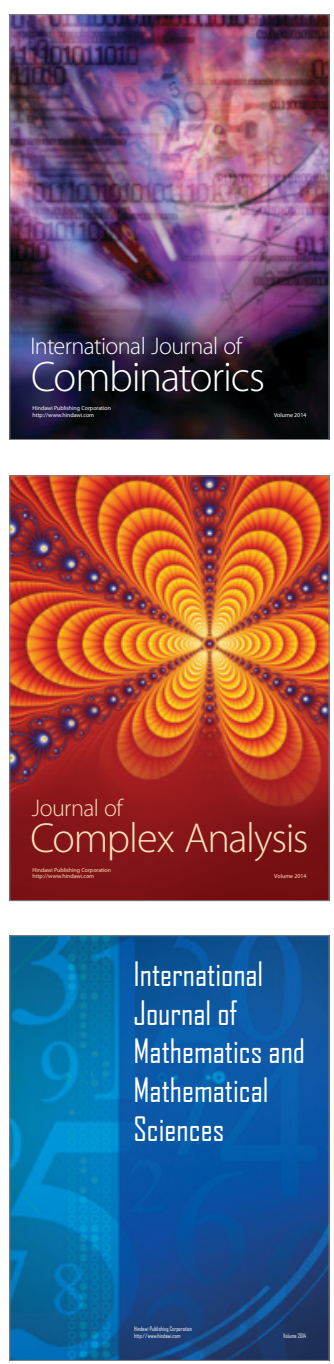
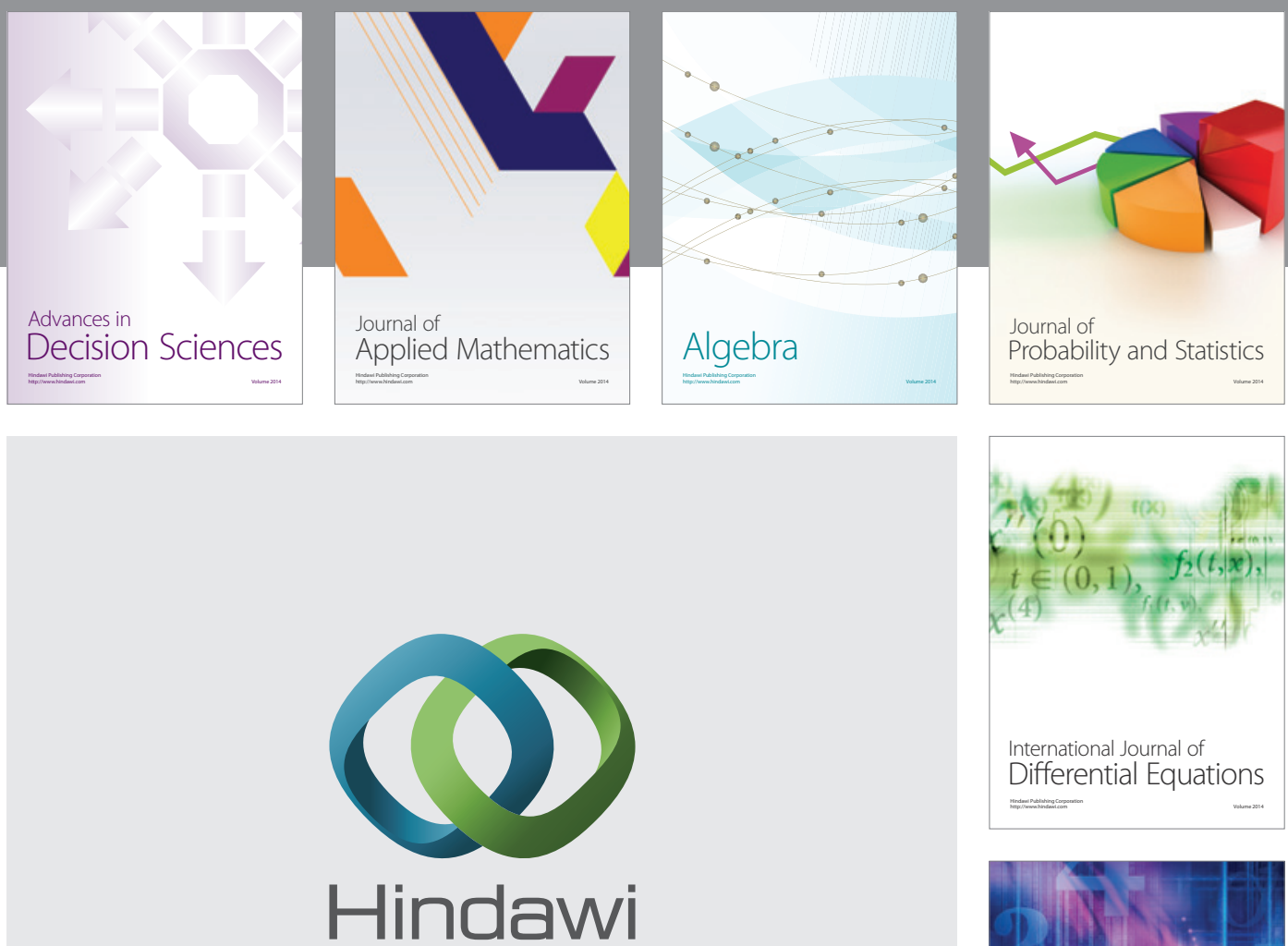

Submit your manuscripts at http://www.hindawi.com
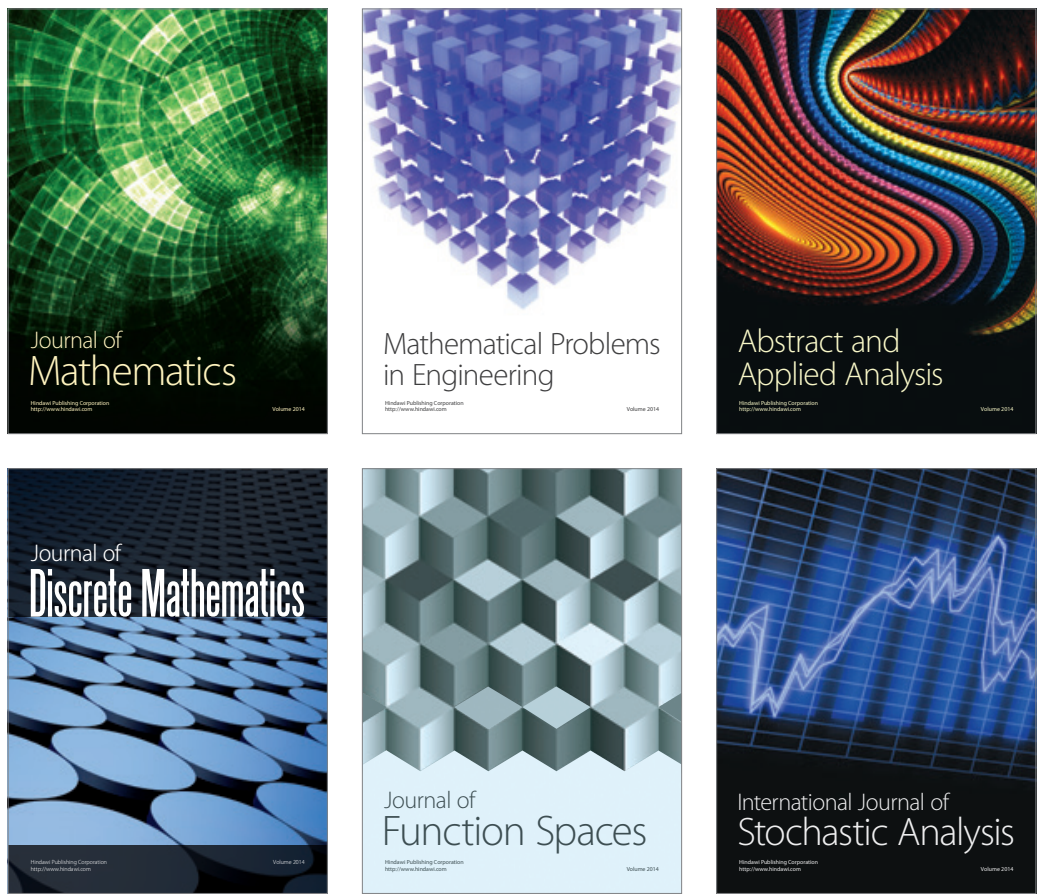

Journal of

Function Spaces

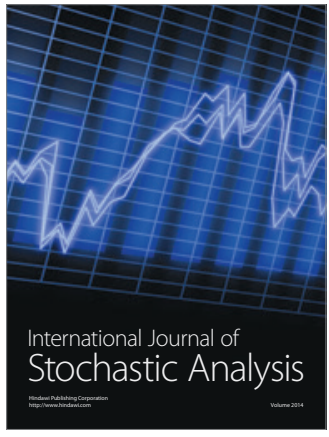

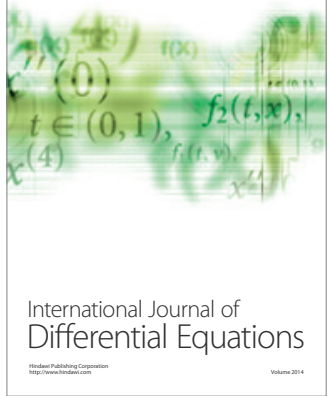
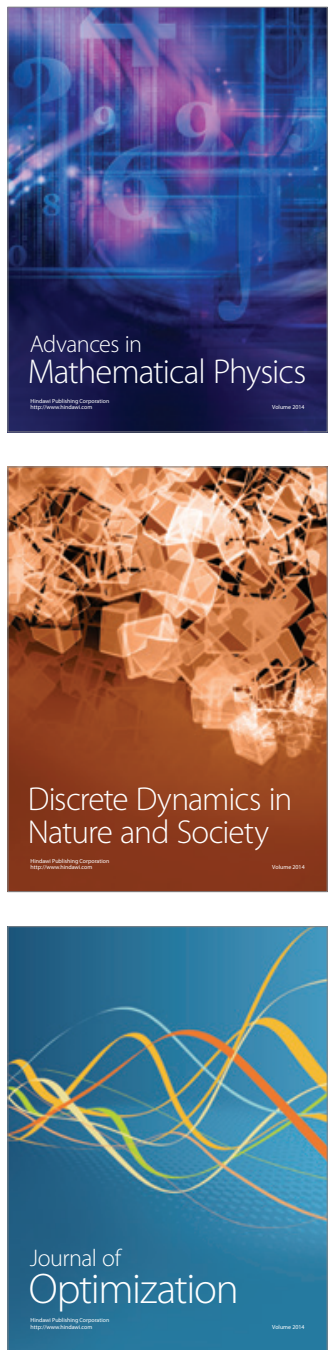